\title{
Increasing Minority Participant Enrollment into a Cancer Family Registry: The Cancer Genetics Network
}

\author{
Deborah J. Bowen $^{d}$ Thuy Vua, ${ }^{a}$ Carol Kasten-Sportes ${ }^{c}$ \\ ${ }^{a}$ Fred Hutchinson Cancer Research Center and ${ }^{b}$ University of Washington, Seattle, Wash.; ${ }^{c}$ National Cancer Institute, \\ Bethesda, Md.; ${ }^{d}$ Social and Behavioral Sciences, School of Public Health, Boston University, Boston, Mass., USA
}

In 1998, the National Cancer Institute funded an innovative national Cancer Genetics Network (CGN) which provides participating researchers access to a breadth of research data not currently available to most individual cancer genetics programs. The CGN is composed of eight centers around the nation which are linked to a central informatics center. The Network supports collaborations to investigate the genetic basis of cancer susceptibility, explore mechanisms to integrate this new knowledge into medical practice, and identify ways to address associated psychosocial, ethical, legal, and public health issues [1]. As of May 2002, the CGN contained data on 15,007 participants and 241,948 family members. The majority of CGN participants were of Non-Hispanic White/Caucasian ethnicity (90\%), with few numbers of Hispanic (4\%), Black (3\%), Asian (1\%), and other ethnicities (2\%). These participation figures did not match those of the general public, nor did they match the catchment areas of the participating sites in the CGN.

The investigators and NIH staff reviewed the disparities in recruitment yield 4 years after the registry funding period began, and decided to take action to improve recruitment of minority participants. The investigators decided to conduct research into minority recruitment, focusing on ways to enhance ethnic minority participation into the registry. The sites were charged with two goals: to identify new recruitment sources appropriate to the locale and eligibility requirements of each site, and/or to design and implement studies at each site to test the usefulness of new recruitment strategies, processes, or incentives. A published paper came out of this initial effort to review the literature on this topic [2], as well as a series of ideas and processes to test within the CGN sites. The research team formed working groups, based on scientific expertise located at each center, populations existing in the catchment area of each site, and interest in participating in an effort in minority recruitment. Each of the working groups focused on a package of a different ethnic minority population and a different strategy. The centers at Duke and Georgetown Universities conducted a randomized trial of a recruitment brochure in which the content for potential African American enrollees to the CGN was targeted and enhanced based on formative research with the targeted population. Investigators at the University of Pennsylvania tested the offering of personal risk feedback as an incentive to join CGN, adding questions to the standard CGN survey to capture reactions to the feedback. Efforts at Johns Hopkins focused on working with African American sororities as community contacts to enhance enrollment into the network. The group at the University of Texas conducted a randomized trial to test the effects of a targeted print media product, a magazine, to enhance recruitment of Hispanic participants into the CGN. The collaborative study conducted by investigators at both the University of California Irvine and the Fred Hutchinson Cancer Research Center

\section{KARGER \\ Fax +4161306 1234 \\ E-Mail karger@karger.ch}

www.karger.com
(C) 2008 S. Karger AG, Basel

$1422-2795 / 08 / 0114-0191 \$ 24.50 / 0$

Accessible online at:

www.karger.com/cmg
Deborah Bowen

Social and Behavioral Sciences

Boston University, 715 Albany Street T2W

Boston, MA 02118 (USA)

Tel. +1 617638 5205, Fax +1 617638 4483, E-Mail dbowen@bu.edu 
was a randomized trial of a pan-Asian message of welcome to the CGN to increase Asian recruitment.

The papers in this focused issue present the results of these efforts by working group. Each manuscript describes an enhanced recruitment process or procedure and presents data on its effectiveness. In addition, most of the project teams collected data outside of the standard CGN enrollment data to enable a greater understanding of the recruitment process for minority individuals.

Overall enrollment of minority participants into the CGN was enhanced by these minority recruitment efforts. The enrollment of Black, Hispanic, and Asian participants over the period of the minority recruitment efforts increased steadily, from 1,315 participants to 1,942 participants. The efforts of the specific studies focused on minority participants drove this change.

This collaborative activity represented our first attempt to identify issues that would produce lower enrollment and to enhance the attractiveness of participating in a cancer registry for minority individuals. We hope that the data collected and presented here will be useful to improving recruitment yields in future research projects of this type. We also find this type of multidisciplinary collaboration useful in creating a setting where issues of race, recruitment, and scientific discussions can occur and can lead to new data efforts. We hope to continue this type of work in the future.
References
2 Hughes C, Peterson S, Ramirez A, Gallion K, McDonald P, Skinner C, Bowen DJ: Minority recruitment in hereditary breast cancer research. Cancer Epidemiol Biomarkers Prev 2004;13:1146-1155.
Hiatt R, Nayfield S: The Cancer Genetics Network: recruitment results and pilot studies. Community Genet 2003;6:171-177.
Anton-Culver H, Ziogas A, Bowen D, Finkelstein D, Griffin C, Hanson J, Isaacs C, Kasten-Sportes C, Mineau G, Nadkarni P, Rimer B, Schildkraut J, Strong L, Weber B, Winn D, 